Técnicas básicas de electroencefalografía: principios y aplicaciones clínicas

\title{
Basic techniques of electroencephalography: principles and clinical applications
}

\author{
F. Ramos-Argüelles, G. Morales, S. Egozcue, R.M. Pabón, M.T. Alonso
}

\section{RESUMEN}

El electroencefalograma es una técnica de exploración funcional del sistema nervioso central (SNC), de relativa antigüedad, pero que aún hoy día sigue siendo una herramienta de gran ayuda para el clínico en el diagnóstico y seguimiento de algunas patologías, como pueden ser la epilepsia, las encefalopatías, alteraciones del estado de conciencia, infecciones del SNC, etc. Es, por otro lado, una herramienta diagnóstica con aplicaciones que están en plena expansión, en combinación con otras técnicas neurofisiológicas, como es en el campo del estudio y diagnóstico de la patología de sueño (polisomnografía, test de latencias múltiples del sueño...) y la monitorización intraoperatoria junto con los potenciales evocados somatosensoriales. Se describen en este trabajo cuáles son los fundamentos técnicos de la electroencefalografía, haciendo especial hincapié en sus principales aplicaciones clínicas y en las perspectivas del futuro.

Palabras clave. Electroencefalografía. Utilidades clínicas. Técnicas básicas. Epilepsia. Patrones EEG.

\begin{abstract}
The electroencephalogram is a technique for the functional exploration of the central nervous system (CNS). It is a relatively old technique but even today it continues to be a tool of great assistance to the clinician in diagnosing and treating certain pathologies, such as epilepsy, encephalopathies, alterations to the state of consciousness, CNS infections, etc. On the other hand, it is a diagnostic tool whose applications are expanding in combination with other neurophysiological techniques, such as in the field of the study and diagnosis of sleep pathology (polysomnography, multiple sleep latency test...) and in intraoperative monitoring together with somasensory evoked potentials. This article describes the basic techniques of electroencephalography, with special emphasis on its main clinical applications and on future perspectives.
\end{abstract}

Key words. Electroencephalography. Clinical units. Basic techniques. Epilepsy. EEG patterns.
Servicio de Neurofisiología Clínica. Hospital Virgen del Camino. Pamplona.

\section{Correspondencia}

Gonzalo Morales Blánquez

Servicio de Neurofisiología Clínica

Hospital Virgen del Camino

Irunlarrea, 4

31008 Pamplona

E-mail: gonzalo.morales.blanquez@cfnavarra.es 


\section{INTRODUCCIÓN: BASES Y ASPECTOS TÉCNICOS}

La electroencefalografía es una técnica de exploración funcional del sistema nervioso central (SNC) mediante la cual se obtiene el registro de la actividad eléctrica cerebral en tiempo real.

En 1929 Hans Berger acuñó el término "electroencefalograma», en abreviatura EEG, para describir el registro de las fluctuaciones eléctricas en el cerebro captadas por unos electrodos fijados al cuero cabelludo.

El origen de la señal eléctrica está en las células piramidales de la corteza cerebral. Cada una de dichas neuronas constituye un diminuto dipolo eléctrico, cuya polaridad depende de que el impulso a la célula sea inhibitorio o excitatorio. Para poder recoger y registrar una señal de la actividad eléctrica en cada región cerebral a través de la superficie craneal se colocan electrodos que captan la diferencia de potencial entre ellos. La EEG realiza el estudio y análisis de los campos eléctricos cerebrales (topografía, polaridad y su variación espacial temporal) mediante la amplificación de la diferencia de potencial entre los electrodos receptores de la señal. Pueden estar situados en el cuero cabelludo (EEG estándar), en la superficie cortical (EEG cortical) o intracerebrales (EEG de profundidad).

El EEG estándar es una exploración indolora, no invasiva, de bajo coste, que puede ser de gran utilidad en la práctica clínica. Se realiza colocando electrodos de superficie adheridos al cuero cabelludo por un gel conductor. Se posicionan de acuerdo al sistema internacional $10-20^{1,2}$. Cada derivación o canal de registro, mide la diferencia de voltaje entre dos electrodos (uno es el activo y otro el de referencia). Lo habitual es que se usen de 16 a 24 derivaciones en cada montaje ${ }^{3,4}$.

Los distintos pares de electrodos se combinan constituyendo los montajes. Hay dos tipos básicos de montajes: bipolar (transversal y longitudinal) y monopolar (o referencial). El bipolar registra la diferencia de voltaje entre dos electrodos colocados en áreas de actividad cerebral, mientras que el monopolar registra la diferencia de potencial entre un electrodo ubicado en una zona cerebral activa y otro colocado sobre un área sin actividad o neutra (por ejemplo el lóbulo de la oreja); o bien, la diferencia de voltaje entre un electrodo colocado en una zona activa y el promedio de todos o algunos de los electrodos activos.

El uso de electrodos invasivos permite estudiar en detalle áreas cerebrales en ocasiones de difícil acceso como la superficie mesial del lóbulo temporal (electrodos nasofaríngeos, electrodos esfenoidales, del foramen oval, temporales anteriores, etc.).

Actualmente los aparatos utilizan amplificadores digitales. La señal analógica está en completo desuso, por las grandes ventajas que el EEG digital aporta entre las que destacan la facilitación de la adquisición, análisis y almacenamiento de la señal y la posibilidad de modificar, tanto durante la grabación, como a posteriori, parámetros como filtros, sensibilidad, tiempo de registro y montajes (Fig. 1).

Para la obtención del trazado, se requiere que el paciente esté relajado, en un ambiente de semipenumbra y con los ojos cerrados. Deberá dormir lo habitual la noche previa a la realización de la prueba, excepto en el caso de que se vaya a realizar un EEG con privación de sueño para el cual se precisan de 12 a 24 horas de vigilia previas. No es necesario suspender la medicación habitual aunque sí debe anotarse para una posterior interpretación adecuada del trazado. La suspensión de la medicación antiepiléptica sólo se efectúa cuando se desea registrar una crisis en vídeo en paciente hospitalizado. La duración que ha de tener un EEG estándar es de al menos 30 minutos de registro e incluye técnicas de activación, principalmente la fotoestimulación intermitente y la hiperventilación. Estas técnicas están dirigidas a provocar o intensificar la aparición de anomalías en la actividad cerebral. 


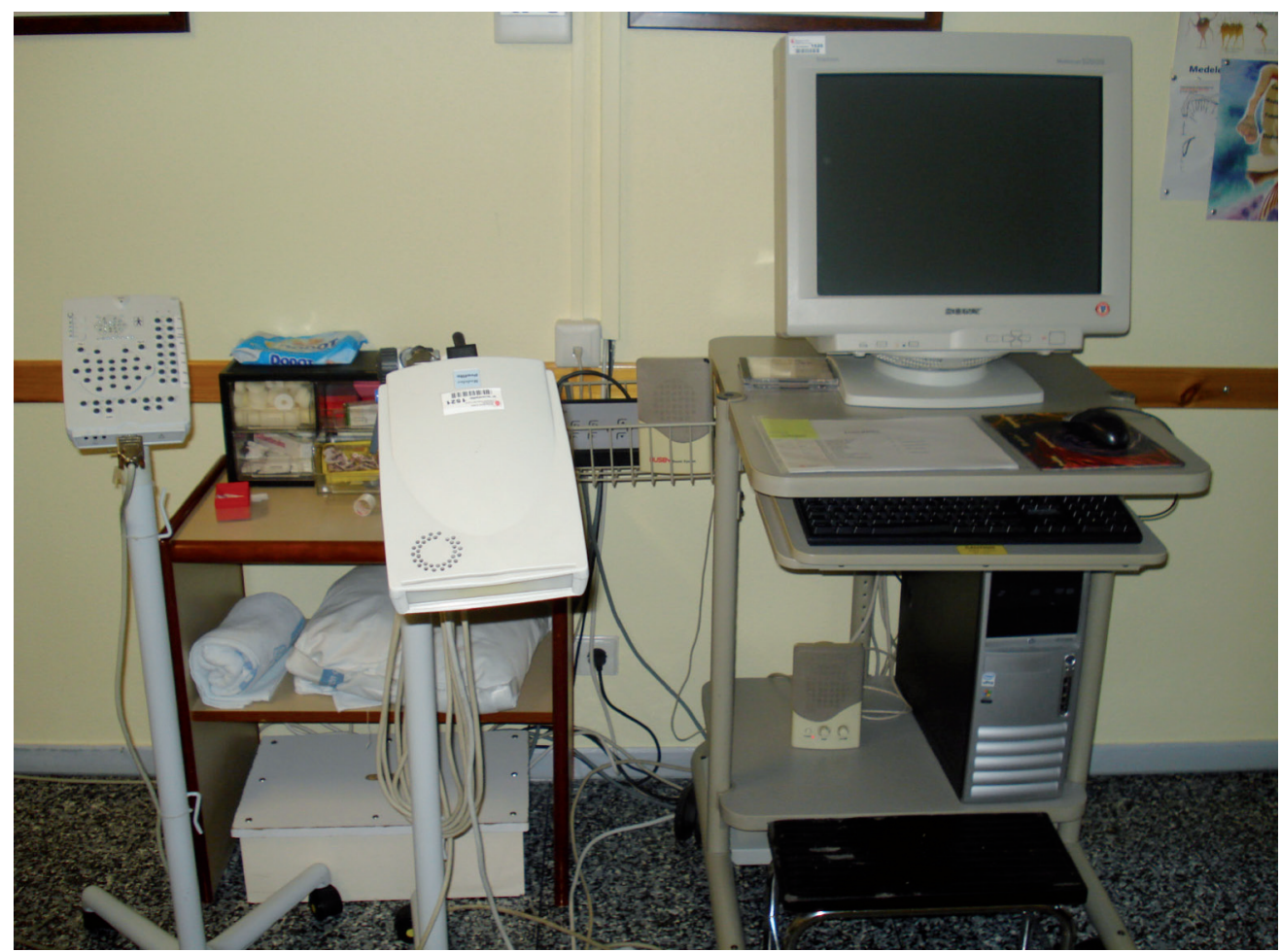

Figura 1. Equipo de electroencefalografía digital.

La hiperventilación consiste en que el paciente debe respirar por la boca de forma regular y profundamente durante unos 3 minutos. Está contraindicada en algunos casos (edad muy avanzada, enfermedad cardíaca o pulmonar severa, infarto agudo de miocardio, hemorragia cerebrovascular reciente, hipertensión arterial no controlada).

Durante la fotoestimulación luminosa intermitente se aplican estímulos luminosos a diversas frecuencias. Se estimula primero con los ojos abiertos, y tras 5 segundos se le pide al paciente que cierre los ojos. Se comienza ascendiendo desde $1 \mathrm{~Hz}$ hasta $20 \mathrm{~Hz}$, y después se desciende desde 60 hasta $25 \mathrm{~Hz}$. La duración aproximada es de 6 minutos.

El objetivo es determinar el umbral de frecuencia de estimulación tanto inferior como superior que ocasiona una respuesta fotosensible del paciente. Las frecuencias más efectivas se encuentran entre los 15 y los $20 \mathrm{~Hz}$. La estimulación de una determinada frecuencia debe detenerse cuando se provoca una respuesta motora generalizada. El estímulo es más eficaz en el momento de cerrar los ojos. La estimulación binocular es más eficaz que la monocular. La fotosensibilidad es claramente hereditaria. Se asocia con las epilepsias generalizadas, particularmente aquellas que tienen un fuerte componente hereditario. Afecta más a mujeres y hay un rango de edad (entre los 5 y los 15 años) en el que se da con más frecuencia.

Otras técnicas de activación de anomalías sobre todo de tipo epiléptico son el sueño y privación de sueño.

La sedación, al alterar profundamente la actividad eléctrica cerebral, no se debe realizar salvo en aquellos casos de pacientes que no cooperen en la realización del registro (como en el caso de pacientes con 
retraso mental severo, con alteraciones conductuales, etc. $)^{5}$.

Actualmente se han desarrollado además de las comentadas, múltiples técnicas que han permitido una evaluación neurofisiológica más sofisticada de la función cerebral como son la monitorización vídeoEEG, EEG ambulatorio, telemetría, mapeo cerebral y la electrocortigrafía.

\section{APLICACIÓNES CLÍNICAS DE LA ELECTROENCEFALOGRAFIA}

En el momento actual el EEG está indicado fundamentalmente en cuadros neurológicos paroxísticos, alteraciones del nivel de conciencia, deterioro de funciones superiores y en la evaluación de la maduración cerebral en recién nacidos y prematuros.

Hay que resaltar que la información que aporta el EEG no está limitada al campo de la epilepsia. Es posible obtener una gran cantidad de información clínicamente relevante del EEG en patologías no epilépticas, en el coma y en la muerte cerebral, las migrañas $^{6}$, los accidentes isquémicos cerebrales $^{7,8}$, las encefalopatías metabólicas (encefalopatía hepática, eclampsia, hipo e hipercalcemias, hiponatremia, hipertiroidismo.... $)^{9}$, las encefalopatías mixtas, los traumatismos craneoencefálicos, las infecciones del sistema nervioso central (entre ellas la encefalitis, la enfermedad de Creutzfeldt-Jakob, la panencefalitis esclerosante subaguda...) y los tumores intracraneales $^{10}$.

\section{Epilepsias}

En el campo de la epilepsia, la ayuda que el EEG proporciona es evidente. Es decisivo para el diagnóstico, para la clasificación de la crisis o síndrome epiléptico del paciente, para el control evolutivo del paciente y ayuda en la decisión de supresión del tratamiento. Hay que distinguir entre las actividad epileptiforme intercrítica (puntas, punta-onda, poli punta y poli punta-onda) y la actividad crítica que se registra durante las crisis.
Antes de valorar el EEG es preciso tener en cuenta que hasta el $50 \%$ de los pacientes epilépticos pueden tener un EEG intercrítico normal y que un $2 \%$ de la población sana tiene puntas en el EEG. Incluso el EEG crítico puede ser normal en el $40 \%$ de las crisis parciales simples y en el $10 \%$ de las crisis parciales complejas.

Por todo ello, es de gran utilidad realizar activaciones (hiperpnea, sueño, privación de sueño, fotoestimulación), registros prolongados de 24 horas o más o un registro simultáneo y sincronizado de vídeo y EEG, que permitirá ponderar correctamente los fenómenos clínicos y electroencefalográficos así como aumentar las posibilidades de diagnóstico ${ }^{11-13}$.

\section{Epilepsias en el niño}

La mayor parte de las epilepsias aparecen en la infancia. El EEG es fundamental en el diagnóstico y para la clasificación del síndrome epiléptico que sufre el paciente.

Dependiendo de la patología se pueden encontrar diversos patrones electroencefa$\operatorname{lográficos}{ }^{14}$ :

- Convulsiones febriles. Enlentecimiento generalizado hasta $72 \mathrm{~h}$ después de la crisis. Un ritmo theta posterior a 4-7 $\mathrm{Hz}$ puede indicar recurrencia de la crisis.

- Síndrome de West. Hipsarritmia, que es la mezcla anárquica de ondas lentas de gran amplitud, ondas agudas y puntas. Durante el sueño lento los paroxismos se hacen más síncronos y pueden aparecer separados por periodos de depresión de la amplitud, de forma pseudoperiódica; por otro lado los espasmos se puede presentar de dos formas: atenuación paroxística del voltaje o como una desincronización del trazado, constituida por ritmos rápidos.

- Síndrome de Lennox-Gastaut. Actividad de base lentificada. Paroxismos generalizados de punta-onda lenta a $2-2,5 \mathrm{~Hz}$, con predominio frontal. Puede haber actividad focal o multifocal 
punta-onda. En el sueño No REM los paroxismos anteriormente descritos (que en sueño cambian de morfología, pasando a ser polipunta-onda lenta) tienen una mayor tendencia a la sincronización, con un incremento de las descargas, que disminuyen en el sueño REM. Los ritmos propios del sueño se entremezclan con descargas de puntas rítmicas.
- Epilepsia con ausencias infantiles. Paroxismos generalizados de puntaonda a $3 \mathrm{~Hz}$ de inicio y fin brusco. La duración de las ausencias oscila entre 5-15 segundos. En el sueño lento las descargas de punta-onda son de menor duración y a veces la punta-onda adquiere morfología de polipunta-onda. Gran activación con la hiperventilación (Fig. 2).

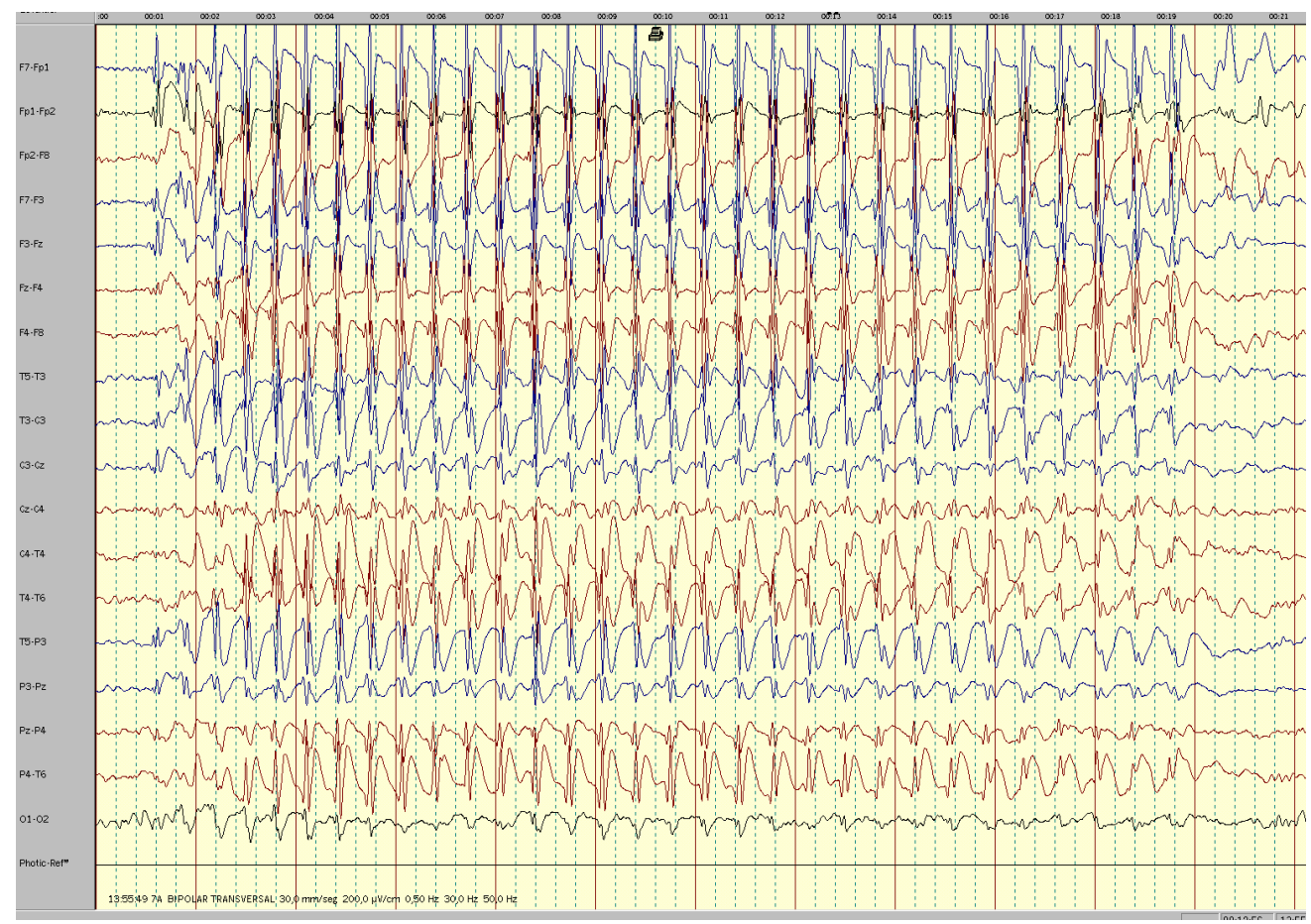

Figura 2. Crisis de ausencia típica (montaje bipolar transversal).

- Epilepsia con crisis tónico-clónicas generalizadas en la infancia. Paroxismos punta-onda bilaterales y síncronos en vigilia y que son activados por el sueño. También pueden ser como generalización de una crisis parcial.

- Epilepsia parcial benigna con paroxismos rolándicos. Paroxismos focales constituidos por puntas centro-temporales, que generalmente se sigue de una onda lenta. Pueden extenderse de un hemisferio a otro. Se activan con el sueño.

- Epilepsia parcial benigna con paroxismos occipitales. Punta-onda lenta en regiones occipito-temporales posteriores, uni o bilaterales. Se bloquea con la apertura ocular. Aumentan con el sueño lento (en ocasiones adquieren aspecto de punta-onda o polipunta-onda generalizadas) (Fig. 3). 


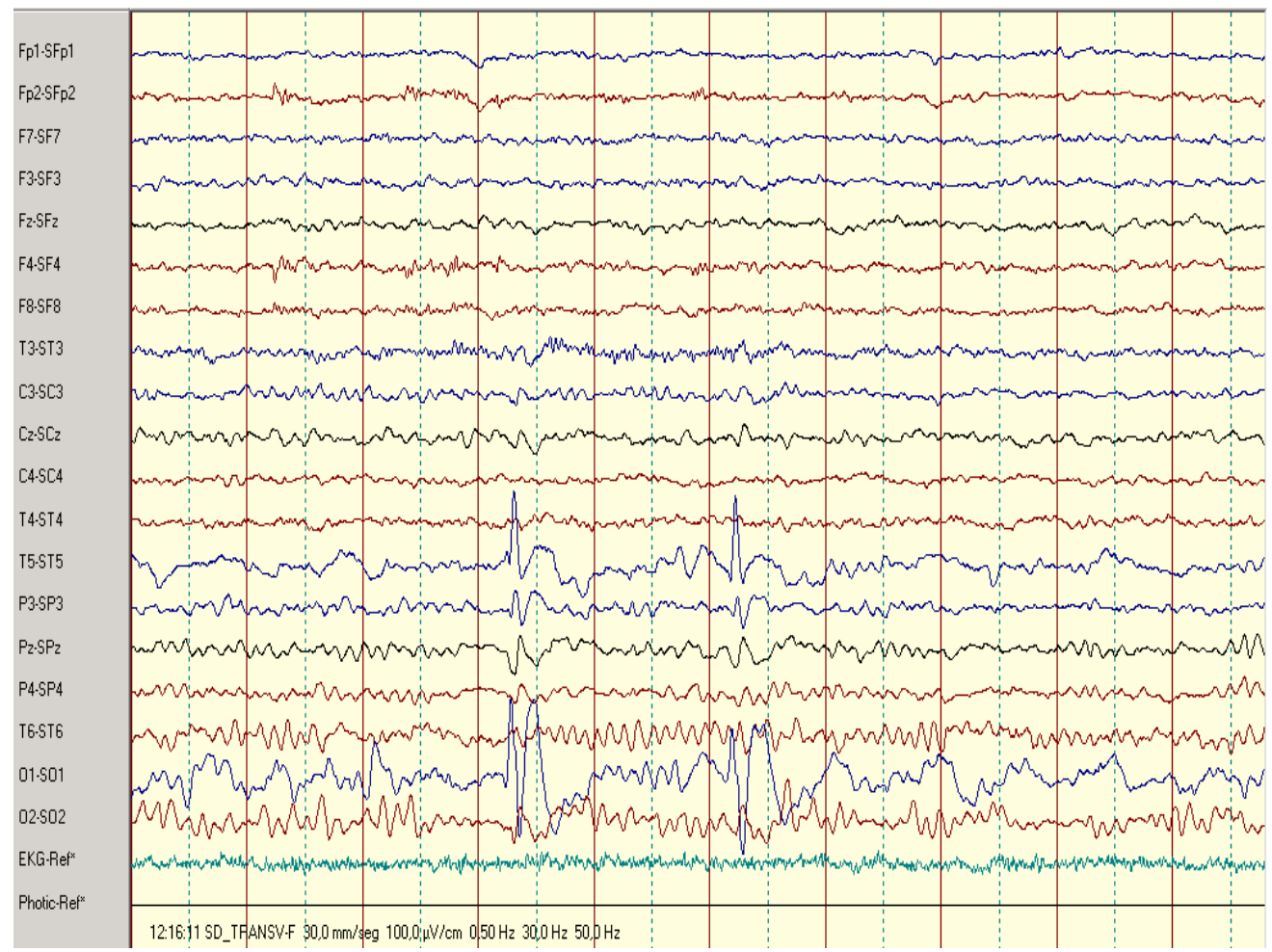

Figura 3. Actividad punta-onda en región posterior izquierda, montaje referencial (source).

- Epilepsia parcial idiopática con puntas frontales. Paroxismos frontales uni o bilateral más frecuentes. A veces con generalización secundaria. Más frecuente durante el sueño o al despertar.

- Síndrome de Landau-Kleffner. Anomalías paroxísticas focales o multifocales, de puntas y punta-onda de gran amplitud, uni o bilaterales, con predominio en regiones temporales. Los paroxismos se generalizan durante el sueño lento.

- Epilepsia con punta-onda continua durante el sueño lento. En vigilia, paroxismos de punta-onda frontocentrales o centro-temporales. Durante todo el sueño lento, aparece actividad punta-onda lenta continua, bilateral y difusa, que ocupa más del 85\% del trazado de sueño no REM. Esta actividad desaparece en el sueño REM, quedando escasas descargas de punta-onda difusa y/o paroxismos focales aislados de predominio frontal.

- Epilepsia mioclónica juvenil. Complejos polipunta-onda bilaterales y síncronos, a más de $3 \mathrm{~Hz}$. En el $30-40 \%$ de los casos aparece fotosensibilidad, que es más frecuente en el sexo femenino (la acción conjunta de la fotoestimulación intermitente y el cierre palpebral aumenta esta proporción) ${ }^{15}$.

\section{Epilepsia del adulto}

Algunos tipos de epilepsia de la infancia descritos anteriormente, como son las parciales secundarias, algunas epilepsias generalizadas primarias (epilepsia mioclónica juvenil) y las epilepsias generalizadas secundarias (síndrome de Lennox-Gastaut) pueden persistir en la edad adulta. No obs- 
tante, la epilepsia parcial es el tipo de epilepsia que se inicia en el adulto con mayor frecuencia.

La epilepsia del lóbulo temporal es el síndrome epiléptico parcial más frecuente del adulto. En él se han descrito distintos patrones interictales: la punta temporal anterior es la alteración intercrítica más típica de la epilepsia del lóbulo temporal. Se activa con el sueño y con la privación de sueño. La actividad delta rítmica intermitente temporal se relaciona con crisis parciales complejas originadas en el lóbulo temporal. Durante las crisis se registra una atenuación de la actividad de fondo, seguida de una actividad rítmica theta.

La epilepsia del lóbulo frontal es el segundo síndrome parcial más frecuente en el adulto. Los patrones que encontramos son descargas focales de ondas agudas, puntas, polipuntas, punta-onda, polipuntaonda o actividad de alta frecuencia y bajo o medio voltaje. Es una zona de habitual propagación de la actividad epileptiforme al lado contralateral, por lo que es común encontrar descargas de punta-onda y polipunta-onda generalizadas o bifrontales. Por lo tanto el EEG interictal es mucho menos sensible y específico para la localización de la zona epileptógena que en la epilepsia del lóbulo temporal. Con frecuencia el único cambio ictal en estas crisis es una actividad rítmica theta o delta de corta duración, generalmente enmascaradas por artefactos musculares. Suelen aparecer durante el sueño.

Por último las epilepsias parietales, caracterizadas por una rápida propagación de la actividad epileptiforme hacia el lóbulo frontal y temporal; y las epilepsias occipitales con una también rápida propagación de las descargas hacia el lóbulo temporal. En las epilepsias parietales y occipitales existen las mismas limitaciones que las comentadas anteriormente en los patrones interictales ${ }^{16,17}$.

\section{Estatus epiléptico}

El estatus epiléptico (EE) es una urgencia neurológica que consiste en la re- currencia de crisis epilépticas sin recuperación de la consciencia entre ellas, o la aparición de una crisis epiléptica continua durante más de 30 minutos. Se puede clasificar en EE generalizado convulsivo o EE no convulsivo.

El EEG es de indudable utilidad en el diagnóstico y seguimiento de pacientes con sospecha de encontrarse en estatus epiléptico, más aún en el caso del estatus epiléptico no convulsivo.

En el EE generalizado convulsivo el EEG pasa por varias fases. Inicialmente aparecen patrones ictales de crisis generalizadas separados por segmentos de fondo con ondas lentas generalizadas. Posteriormente desaparece la actividad lenta entre las crisis dando lugar a la convergencia de las crisis de manera sucesiva. Finalmente aparece la actividad ictal continua propia del EE: patrón continuo de puntas, punta-onda, o polipuntas entremezcladas con ondas lentas rítmicas (1-3 Hz). Esta actividad ictal continua va siendo interrumpida por períodos de atenuación de la actividad eléctrica.

Esta actividad ictal es sustituida por patrones periódicos de descargas epileptiformes generalizadas o lateralizadas, constituidos por puntas, ondas agudas y polipuntas. Estas descargas periódicas pueden permanecer en el EEG durante 3-5 días hasta ser sustituidas por descargas semiperiódicas. Por último, quedará una actividad de fondo lenta de manera continua.

En el EE no convulsivo el EEG puede ser de gran utilidad ya que en muchas ocasiones el paciente presenta como clínica un cuadro confusional sin que se sospeche la presencia de un cuadro epiléptico. En el EEG se pueden distinguir tres patrones principales:

1. Tipo ausencia: descargas de puntaonda lenta a $3 \mathrm{~Hz}$ generalizada.

2. Tipo parcial complejo: patrones ictales continuos o recurrentes de origen temporal (actividad rítmica de 4 a 7 $\mathrm{Hz}$ en región temporal, que crecen y decrecen en amplitud y frecuencia), frontal (punta-onda generalizada o parasagital sincrónica), occipital o parietal. 
3. Tipo parcial simple: descargas epileptiformes a $1 \mathrm{~Hz}$ que puede desaparecer de manera intermitente ${ }^{18-19}$.

\section{Coma y muerte cerebral}

En la valoración dinámica del estado de conciencia que proporciona el EEG son varios los aspectos esenciales que se deben analizar de cara al pronóstico del paciente:

- El grado de lentificación de la actividad cerebral: una menor frecuencia se suele relacionar con el grado de reducción del nivel de conciencia y un peor pronóstico.

- Actividad cerebral continua o discontinua. Una actividad cerebral continua va a favor de un mejor pronóstico.

- Presencia de actividad epileptiforme. Empeora el pronóstico. Se pueden ver puntas, ondas agudas, brotes de punta-onda, que pueden estar o no asociados a crisis epiléptica o estatus no convulsivo.

- Reactividad a estímulos: a medida que el coma es más profundo la actividad cerebral es menos reactiva a cualquier tipo de estímulo (doloroso, auditivo, etc.)

- Presencia de patrones de sueño (husos, ondas de vértex, complejos k...) son más infrecuentes conforme el coma es más profundo.

- La depresión progresiva del voltaje es sugestiva de afectación del tronco encefálico y por tanto, supone un peor pronóstico. Voltajes inferiores a 20 microvoltios son precursores del silencio electrocerebral ${ }^{20}$.

Otros datos de gran interés que aporta el EEG en el pronóstico de pacientes con alteración del nivel de conciencia son ${ }^{21,22}$.

- Brotes intermitentes de actividad delta frontal (FIRDA) en adultos o posterior (OIRDA) en niños. Se asocian a encefalopatías metabólicas, tóxicas o hipóxicas, lesiones supratentoriales, lesiones subcorticales. Es muy sugerente de las fases tem- pranas del coma, representando un signo inicial de disfunción del sistema de alerta.

- Patrón alfa difuso. Presencia de actividad en rango alfa $(8-13 \mathrm{~Hz})$ de distribución difusa y de manera continua, de predominio en región anterior, sin modificación por la apertura de ojos. Puede presentarse en rango theta $(4-7 \mathrm{~Hz})$, teniendo el mismo significado. Los pacientes con este patrón habitualmente fallecen o permanecen en estado vegetativo.

- Ondas trifásicas. Típico de la encefalopatía hepática y de otras encefalopatías matabólicas. También puede observarse en la enfermedad de Creutzfeld-Jacob y otras demencias.

- Descargas periódicas epileptiformes lateralizadas (PLEDS). De horas o días de duración. Habitualmente en relación a accidente cerebrovascular agudo. También en otras situaciones: neoplasias, encefalitis, estatus epiléptico, encefalopatía isquémico-anóxica tras parada cardiaca. En la mayoría de los casos los PLEDS se asocian con crisis epilépticas (crisis motoras o estatus epiléptico parcial). En pacientes con síndrome confusional o estuporoso y PLEDS es difícil diferenciar entre estatus epiléptico no convulsivo y estupor no epiléptico debido a la causa primaria (puede ayudar a diferenciarlos la administración intravenosa de benzodiazepinas).

- Patrón brote supresión. Brotes de actividad aguda o lenta de alto voltaje intercalados con períodos de supresión de la actividad eléctrica. Aparece típicamente en la encefalopatía isquémica-anóxica. La evolución de este patrón es habitualmente la muerte cerebral o el estado vegetativo persistente. Algunos anestésicos (midazolam, propofol) generan este patrón de forma reversible. 
- Patrón de supresión generalizada o EEG isoeléctrico. No hay indicios de actividad EEG con una amplificación de 2 microvoltios $/ \mathrm{mm}$.

En los pacientes con encefalopatía isquémico-anóxica el EEG isoeléctrico a las 24 horas de recobrar la circulación es diagnóstico de muerte cerebral. Sin embargo, el patrón isoeléctrico en sí mismo no es sinónimo de muerte cerebral (aparece también en pacientes con mutismo acinético, hipotermia y en intoxicaciones farmacológicas, con recuperación completa posterior) ${ }^{23,17}$.

\section{Otros patrones electroencefalográficos de interés}

- Encefalitis herpética. Muy frecuentemente se encuentra un patrón muy característico como son los PLEDS. Son ondas agudas y/o puntas, con un intervalo entre ellas de entre 0,5 y 4 segundos, de localización principalmente temporal. En ocasiones, si la afectación es bilateral, estas descargas pueden aparecer en ambos hemisferios denominándose entonces BiPLEDS (Fig. 4).

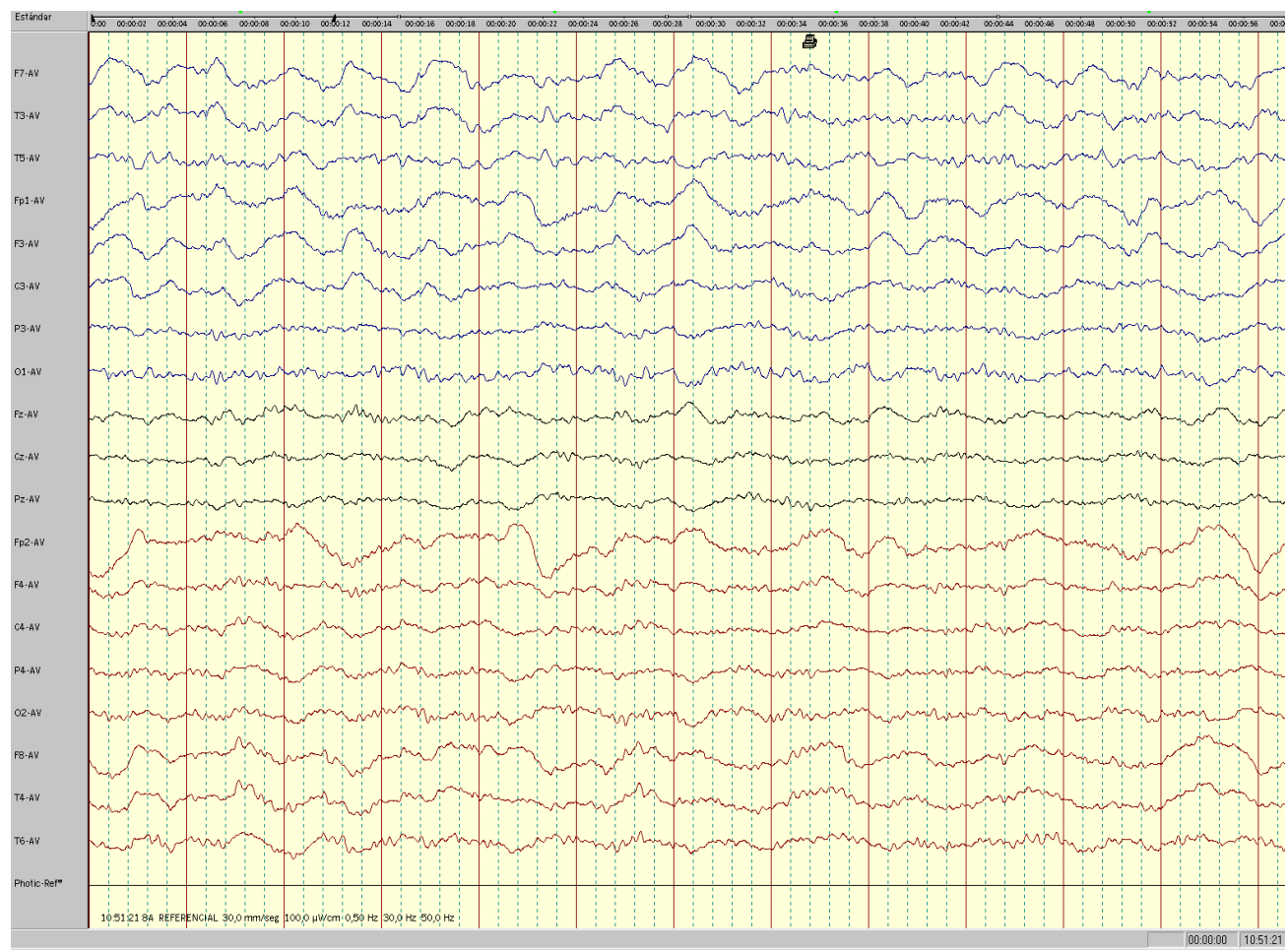

Figura 4. Encefalitis herpética: presencia de ondas agudas y lentas en ambas regiones temporales (montaje referencial).

- Panencefalitis esclerosante subaguda. Se observan las llamadas descargas periódicas generalizadas de intervalo largo (PLIDDS). Son complejos polifásicos, con una mezcla de frecuencias, que incluyen componentes agudos agudos y ondas delta, con una duración de 0,5 a 3 segundos y una recurrencia con intervalos de más de 4 segundos. Reciben el nombre de "complejos de Radermecker". Los complejos pueden ser simétricos y síncronos, aunque en ocasiones son asimétricos. Las descargas pue- 
den aparecer muy temprano en el curso de la enfermedad, cuando los pacientes aún están alerta. A veces, los complejos no aparecen en vigilia o son esporádicos, pero aparecen o se hacen más periódicos durante el sueño.

- Anoxia cerebral e intoxicación por barbitúricos. Puede observarse el mismo patrón descrito anteriormente. En casos de anoxia cerebral los PLIDDS suelen ser de tipo brote-supresión, ya que la actividad eléctrica entre los complejos es de baja amplitud por el daño cortical. En la anoxia cerebral se puede observar también otro patrón, que son las descargas periódicas generalizadas de intervalo corto (PSIDDS). Son ondas agudas, puntas, polipuntas u ondas trifásicas, con un intervalo entre ellas de 0,5-4 segundos, de distribución difusa.

- Encefalopatía hepática: Aparte de una lentificación de la actividad de fondo, encontraremos PSIDDS, concretamente ondas trifásicas de distribución generalizada, aunque con predominio en región frontal. También podemos encontrar este patrón en la encefalopatía urémica.

- Enfermedad de Creutzfeldt-Jakob ${ }^{24,25}$. En las fases inicales el EEG suele mostrar una lentificación difusa del trazado. A los tres meses aparecen complejos periódicos agudos, de morfología bifásica o trifásica, con una frecuencia de $1 \mathrm{~Hz}$ (PSIDDS), no reactivos a estímulos externos y de distribución generalizada simétrica o asimétrica. En ocasiones puede haber un inicio lateralizado en un hemisferio. En fases avanzadas de la enfermedad disminuye la frecuencia de los complejos, aumenta la distancia entre ellos y aparece una actividad cerebral de bajo voltaje. En la variante nueva de encefalopatía por priones, con un curso más lento y que afecta a sujetos jóvenes, el EEG muestra una lentificación difusa, sin complejos periódicos agudos ${ }^{17}$.

\section{EEG en neonatos}

El EEG de un prematuro o de un recién nacido a término difiere mucho del adulto. En general, debemos ser mucho más «permisivos", pues se trata de un cerebro que aún está madurando, por lo que se considera normal encontrar ciertos grados de asimetría o discontinuidad en la actividad cerebral, lentificaciones, o elementos agudos que en otros grupos de edad consideraríamos como patológicos ${ }^{26,27}$.

En neonatos sanos, según la edad gestacional (EG) de los mismos, las características del EEG son las siguientes:

- Actividad de base. Comienza a ser una actividad continua a partir de las 32 semanas de EG. Hasta entonces es normal que se alternen períodos de actividad cerebral con períodos de supresión de la misma. La duración de los períodos inactivos se va reduciendo a medida que es mayor la EG (desde 8 minutos a las 22 semanas de EG hasta 3 segundos de duración de los mismos a las 30 semanas de EG).

- Sincronía. El EEG puede ser asíncrono hasta las 32 semanas de EG.

- Diferenciación sueño-vigilia. A partir de la semana 32 de EG se puede diferenciar la vigilia del sueño. Este último estará constituido por sueño activo (precursor del futuro sueño REM) y sueño tranquilo (futuro sueño no REM).

- Reactividad. El EEG puede ser arreactivo hasta la semana 32 de EG, cuando comienza a reaccionar a estímulos.

Se describen a continuación algunas patologías que podemos encontrar en el neonato prematuro o a término y su correlato electroencefalográfico:

- Hidrocefalia congénita: actividad de base alterada, con abundancia de grafoelementos anormales y crisis eléctricas.

- Agenesia del cuerpo calloso: el trazado puede ser normal. Pueden existir anomalías focales temporales, ondas lentas asíncronas y períodos de atenuación del voltaje. 
- Citomegalia, toxoplasmosis y sífilis congénita: alteraciones de la actividad de base y anomalías focales.

- Hemorragia intraventricular: puntas positivas rolándicas y en vértex, pudiendo haber un patrón brote-supresión.

- Hemorragias subaracnoideas, subdurales, y extradurales: el EEG suele ser normal.

- Infarto hemorrágico periventricular: ondas agudas en región rolándica y en vértex.

- Encefalopatía hipóxico-isquémica: depresión del voltaje y disminución de la frecuencia del EEG. Pueden existir puntas multifocales y patrón de brote-supresión ${ }^{28}$.

- Hiperglicinemia no cetósica: brotesupresión.

- Crisis clónicas focales: frecuente repetición de descargas de puntas focales.

- Crisis tónicas generalizadas: atenuación del voltaje de forma paroxística.

- Mioclonías focales o generalizadas: descargas de puntas y ondas agudas sobre una actividad de fondo deprimida.

- Encefalopatía mioclónica neonatal: brote-supresión ${ }^{29}$.

\section{Patología del sueño}

La patología del sueño es un campo en enorme expansión en el momento actual como prueba el hecho de que cada vez son más los especialistas que se dedican a la investigación y evaluación de la misma y que un gran número de pacientes, bien como trastorno primario, o bien como consecuencia de patologías subyacentes, presentan un trastorno o desestructuración del mismo. Son muchas las patologías que se pueden incluir en esta categoría, y en las que una polisomnografía (con registro simultáneo de video, EEG, actividad muscular en piernas u otras zonas, movimientos respiratorios torácicos y abdominales, flujo aéreo nasal y bucal, saturación de oxígeno, entre otros) o un test de latencias múltiples del sueño (consistente en realizar al paciente una serie de siestas diurnas que nos permitan medir la latencia de inicio del sueño, habiéndonos asegurado mediante una polisomnografía previa de que la calidad del sueño la noche anterior ha sido óptima) tienen mucho que aportar para un correcto diagnóstico y posterior enfoque terapéutico del paciente ${ }^{30}$. Dichas patologías, que aparecen clasificadas en la ICSD-2 ${ }^{31}$ son: insomnio, trastornos respiratorios (como el tan frecuente SAOS), hipersomnias (narcolepsia, síndrome de Klein-Levin...), trastornos del ritmo circadiano, parasomnias (terrores nocturnos, pesadilla, sonambulismo...), trastornos del movimiento relacionados con el sueño (síndrome de piernas inquietas, movimientos periódicos de las piernas, bruxismo), trastornos del sueño asociados a enfermedades médicas (insomnio familiar fatal, reflujo gastroesofágico, fibromialgia, cefaleas, algunas epilepsias que se manifiestan solo o predominantemente durante el sueño, etc.) y las asociadas a enfermedades psiquiátricas.

De todas estas patologías la más común es el insomnio ${ }^{32}$. En este trastorno, en principio no estaría indicada de entrada una PSG nocturna. Únicamente lo estaría cuando el diagnóstico sea dudoso, cuando se sospeche que el origen real del insomnio es otro trastorno del sueño que impida una buena calidad del mismo (como pueden ser el SAOS o el síndrome de piernas inquietas) o cuando se crea que existe un insomnio que vaya asociado a otra patología del sueño. En la PSG de un paciente insomne se aprecia un aumento de la latencia del inicio del sueño, así como un sueño nocturno muy desestructurado, con múltiples despertares y microdespertares.

El segundo trastorno más frecuente es el SAOS, en el que además de la clínica, es imprescindible la realización de una PSG para su diagnóstico, y es muy recomendable a la hora del tratamiento el titular la CPAP con un registro polisomnográfico siempre que sea posible ${ }^{33}$. 
Otro trastorno del sueño, el síndrome de piernas inquietas ${ }^{34}$, tampoco requiere una PSG para su diagnóstico, ya que este ha de ser puramente clínico. Únicamente se recomienda la PSG en caso de duda diagnóstica o de asociación a otro trastorno del sueño (muy común es la coexistencia de SPI con SAOS). En este caso la PSG nos servirá para medir los movimientos periódicos de las extremidades.

En la narcolepsia, y en concreto en aquella que cursa sin cataplejía, es imprescindible para su diagnóstico un TLMS precedido la noche anterior de una PSG. Precisamente en nuestro centro hemos realizado diversos estudios que avalan la utilidad y ayuda de estas pruebas al diagnóstico de la narcolepsia y otras causas de excesiva somnolencia diurna ${ }^{35,36}$.

En las parasomnias (trastorno de conducta durante el sueño REM, terrores nocturnos, sonambulismo), principalmente en aquellas asociadas a otro trastorno, en casos de duda diagnóstica o diagnóstico diferencial con epilepsias, o en niños, es también recomendable la realización de una PSG.

\section{CONSIDERACIONES FINALES}

A nivel del diagnóstico funcional, existen diversas técnicas de diagnóstico que se van desarrollando en los últimos años, tal es el caso de la magnetoencefalografía (MEG). Es una técnica no invasiva de registro de la actividad funcional del cerebro, que se basa en la detección de los campos magnéticos generados por la actividad eléctrica cerebral. Es una técnica inocua para el paciente y no requiere la utilización de electrodos. No obstante, a pesar de sus aparentes ventajas, su principal campo de aplicación, al menos de momento, es el de la investigación, siendo una de sus limitaciones el tremendo coste económico que supone en comparación con el EEG tradicional, así como otras, como es que la MEG solo detecta los campos magnéticos que sean perpendiculares a la corteza cerebral, los cuales son generados por corrientes neuronales orientadas tangencialmente a la corteza. En cambio, aquellos orientados radialmente no generan un campo magnético fuera de la cabeza ${ }^{37,38}$.

Otra técnica que está siendo de gran utilidad para el diagnóstico funcional en el campo de la epilepsia es la combinación del EEG con la resonancia magnética funcional, que proporciona una gran mejoría en relación con el EEG aislado para localizar la región del cerebro en la que se genera la actividad epileptiforme ${ }^{39}$.

No podemos dejar de nombrar otro gran campo de futuro en la neurofisiología clínica, como es la monitorización neurofisiológica intraoperatoria (MIO).

El principal objetivo de esta última es la detección de cualquier daño neurológico tan pronto como sea posible y durante el periodo de la cirugía durante el cual es reversible. Podemos hablar básicamente de dos tipos de técnicas diferentes de MIO: monitorización y mapeo.

La monitorización es el estudio continuo de la integridad funcional de las vías neuronales (nervios periféricos, raíces nerviosas, médula espinal, nervios craneales), para poder identificar lo más precozmente las alteraciones neurofisiológicas que se produzcan permitiendo llevar a cabo una corrección de las mismas.

El mapeo (Mapping) es la identificación puntual y preservación de los tejidos neuronales funcionales. De gran aplicación en la cirugía de la epilepsia ${ }^{40-42}$.

Por último, como ya se ha comentado antes, el campo de la medicina del sueño se encuentra en plena expansión, siendo un terreno multidisciplinar, con participación de múltiples especialistas, como neurólogos, psiquiatras, neumólogos, pediatras, internistas, médicos de familia, otorrinolaringólogos, odontólogos y cirujanos maxilofaciales, entre otros) y en el que la neurofisiología clínica tiene mucho que aportar, no solo en el campo del diagnóstico, con la polisomnografía nocturna, sino también en el tratamiento y seguimiento de estos pacientes.

La electroencefalografía sigue siendo, por tanto, hoy día, una técnica de gran 
utilidad para el clínico, no sólo en el campo de la epilepsia, sino también en otros muchos como se ha comentado en este trabajo. La clave, por tanto, es aportar al clínico una orientación funcional, que unida a la información estructural que aporta la neuroimagen, le sirvan como complemento en el diagnóstico de la patología del paciente.

\section{BIBLIOGRAFÍA}

1. Homan RW, Herman J, Purdy P. Cerebral location of international 10-20 system electrode placement. Electroencephalogr Clin Neurophysiol 1987; 66: 376-382.

2. Myslobodsky MS, Coppola R, Bar-Ziv J, WeinberGER DR. Adequacy of the International 10-20 electrode system for computed neurophysiologic topography. J Clin Neurophysiol 1990; 7: 507-518.

3. Tatum WO, Husal AM, Benbadis SR, Kaplan PW. Normal EEG. En: Tatum WO, Husai AM, Benbadis SR, PW Kaplan editors. Handbook of EEG interpretration. USA: Demos Medical Punlishing 2008: 1-50.

4. Clenney SL, Jonson SM. Electrodes ans their application. En: S. L. Clenney, S.M. Jonson editors. Back to basics: A handbook of EEG technology. California: Beckman, 1983: 5-16.

5. Castillo JL, Galdames D. Aspectos Técnicos del Electroencefalograma. En: Castillo JL, Galdames D, editores. Neurofisiología Clínica. Santiago, Chile: Editorial Mediterraneo 2004: 1-22.

6. BJoRK M, SAND T. Quantitative EEG power and asymmetry increase $36 \mathrm{~h}$ before a migraine attack. Cephalalgia 2008 Sep; 28: 960-968.

7. Finnigan SP, Rose SE, ChalK JB. Contralateral hemisphere delta EEG in acute stroke precedes worsening of symptoms and death. Clin Neurophysiol 2008; 119: 1690-1694.

8. De Vos CC, Van Maarseveen SM, Browers PJ, VAN PUTTEN MJ. Continuous EEG monitoring during thrombolysis in acute hemispheric stroke patients using the brain symmetry index. J Clin Neurophysiol 2008; 25: 77-82.

9. Amodio P, Campagna F, Olianas S, Iannizzi P, MaPELli D, PENzo M et al. Detection of minimal hepatic encephalopathy: normalization and optimization of the psychometric hepatic encephalopathy score. A neuropsychological and quantified EEG study. J Hepatol 2008; 49: $346-353$
10. Niedermeyer E. The clinical Relevante of EEG interpretation. Clin Electroencephalogr 2003; 34: 93-98.

11. Villanueva-Gómez F. Descripción video-EEG de las crisis epilépticas. Rev Neurol 2000; 30 (Supl 1.): S4-S9.

12. Park KI, Lee SK, Chu K, Lee JJ, Kim DW, Nam H. The value of video-EEG monitoring to diagnose juvenile myoclonic epilepsy. Seizure 2009; 18: 94-99.

13. DALY DD. Epilepsy and syncope. En: Daly DD, Pedley TA, eds. Current practice of clinical electroencephalography, $2^{\text {nd }}$ ed. New York: Raven Press 1990: 269-334.

14. Bauer G, Bauer R, Dobesberger J, Unterberger I, Ortler M, Ndayisaba JP et al. Broard sharp waves-an underrecognized EEG pattern in patients with epileptic seizures. J Clin Neurophysiol 2008; 25: 250-254.

15. Rodríguez Barrionuevo AC, Bauzano Poley E. Diagnóstico sindrómico (atlas electro-clínico). En: Rodríguez Barrionuevo AC, Bauzano Poley E editores. Guía práctica para el diagnóstico y tratamiento de la epilepsia en la infancia. Madrid: Editores Médicos S.A., 1995: 147-308.

16. Westmoreland BF. Epileptiform electroencephalographic patterns. Mayo Clin Proc 1996;71: 501-511.

17. Gil-Nagel A, Parra J, Iriarte J, M. Kanner A, editores. Manual de electroencefalografía. Madrid: McGraw-Hill/Interamericana de España, S.A.U., 2002.

18. Teixeira KC, Cendes F, Guerreiro CA, Guerreiro MM. Focal electrical status (FES): a new finding associated with polymicrogyria. J Clin Neurophysiol 2009 Jun; 26: 155-159.

19. Drislane FW, Lopez MR, Blum AS, Schomer DL. Detection and treatment of refractory status epilepticus in the intensive care unit. J Clin Neurophysiol 2008 Aug; 25: 181-186.

20. Oddo M, Carrera E, Claassen J, Mayer SA, Hirsch LJ. Continuous electroencephalography in the medical intensive care unit. Crit Care Med 2009; 37: 2051-2056.

21. WiJdicks EF, HiJdRa A, Young GB, BassetTi CL, WIEBE S. Quality Standards Subcommittee of the American Academy of Neurology. Practice parameter: prediction of outcome in comatose survivors after cardiopulmonary resuscitation (an evidence-based review): report of the Quality Standards Subcommittee of the American Academy of Neurology. Neurology 2006 Jul 25; 67: 203-210. 
22. Chatrian GE, Bergamasco B, Bricolo A, Frost JD JR, PRIOR PF. IFCN recommended standards for electrophysiologic monitoring in comatose and other unresponsive states. Report of an IFCN committee. Electroencephalogr Clin Neurophysiol 1996; 99: 103-122.

23. KAPLAN PW. The EEG in metabolic encephalopathy and coma. J Clin Neurophysiol 2004; 21: $307-318$.

24. Wang PS, Wu YT, Hung CI, Kwan SY, Teng S, Soong BW. Early detection of periodic sharp wave complexes on EEG by independent component analysis in patients with Creutzfeldt-Jakob disease. J Clin Neurophysiol 2008; 25: 25-31.

25. Wieser HG, Schindler K, Zumsteg D. EEG in Creutzfeldt-Jakob disease. Clin Neurophysiol 2006 May; 117: 935-951.

26. Oet MC, Lemmers PM. Brain monitoring in neonates. Early Hum Dev 2009; 85: 77-84.

27. Hellström-Westas L. Continuous electroencephalography monitoring of the preterm infant. Clin Perinatol 2006; 33: 633-647

28. Rafay MF, Cortez MA, De Veber GA, Tan-Dy C, AL-Futais A, Yoon W et al. Predictive value of clinical and EEG features in the diagnosis of stroke and hypoxic ischemic encephalopathy in neonates with seizures. Stroke 2009: 40; 2402-2407.

29. Rodríguez Barrionuevo AC, Bauzano Poley E, Tosina García E, editores. Guía práctica de neurología neonatal-correlación clínico-EEG. Barcelona: César Viguera, editor 1997.

30. García dE GuRTubay. Estudios diagnósticos en patología del sueño. An Sist Sanit Navar 2007; 30 (Supl. 1): 37-51.

31. AASM. The international classification of sleep disorders. Diagnostic and codingmanual. 2. Westchester, IL: AASM; 2005.

32. Schutte-Rodin S, Broch L, Buysse D, Dorsey C, SAteia M. Clinical guideline for the evaluation and management of chronic insomnia in adults. J Clin Sleep Med 2008; 4: 487-504.

33. Task Force Members: Epstein LJ, KRISTo D, Strollo PJ Jr., Friedman N, Malhotra A, Patil
SP et al. Clinical guideline for the evaluation, management and long-term care of obstructive sleep apnea in adults. J Clinical Sleep Med 2009; 5: 263-276.

34. Littner MR, Kushida C, Anderson WM, Bailey D, BERRY RB, HiRshKowitz M et al. Practice parameters for the dopaminergic treatment of restless legs syndrome and periodic limb movement disorder. Sleep 2004; 27: 557-559.

35. Pabón-Meneses RM, Gurtubay-Gálligo IG, RamosArgüelles González F, Gila-Useros L, MoralesBlánquez G, Alonso-Barrasa M. Narcolepsia. una enfermedad infradiagnosticada. Rev Neurol 2008; 47: 547-555.

36. Pabón Meneses RM, Ramos-Argüelles F, García de Gurtubay I, Gila-Useros L, Morales-Blánguez G, González-Rojas L. Utilidad diagnóstica en registros de tests de múltiples latencias del sueño con montaje clásico ampliado en una serie de 253 pacientes. Rev Neurol 2008; 46: 239-251.

37. LOPES DA SILVA FH. What is magnetoencephalography and why it is relevant to neurosurgery? Adv Tech Stand Neurosurg 2005; 30: 51-67.

38. Pastor-Gómez J, Pulido-Rivas P, Sola RG. Revisión bibliográfica sobre la utilidad de la magnetoencefalografía en la epilepsia. Rev Neurol 2003; 37: 951-961.

39. Cunningham CJ, Zaamout Mel-F, Goodyear B, FEDERICo P. Simultaneous EEG-fMRI in human epilepsy. Can J Neurol Sci 2008; 35: 420-35.

40. Pérez Lorensu PJ, Garrido Babío JM, García MaRíN VM. Monitorización neurofisiológica intraoperatoria. En: Gutiérrez-Rivas E, Jiménez MD, Pardo J, Romero M, editores. Manual de electromiografía clínica. Madrid: Ergon 2008: 205-228.

41. Mendiratta A, Emerson RG. Neurophysiologic intraoperative monitoring of scoliosis surgery. J Clin Neurophysiol 2009 Apr; 26: 62-69.

42. Zamel K, Galloway G, Kosnik EJ, Raslan M, Adeli A. Intraoperative neurophysiologic monitoring in 80 patients with Chiari I malformation: role of duraplasty. J Clin Neurophysiol 2009; 26: 70-75. 